\title{
Analyzing the Influencing Factors of Innovation Capability of Economics and Management Postgraduate Students based on Competency Model
}

\author{
Bing Bai $^{1}$, Zhi-qiong Guo ${ }^{2}$ \\ 1) Business School, Jiangsu Normal University, Xuzhou Jiangsu, 221116, China \\ ${ }^{2)}$ Business School, Jiangsu Normal University, Xuzhou Jiangsu, 221116, China
}

\begin{abstract}
With the accelerated process of China into an innovation-oriented economy, training innovative talent is essential. Cultivation of postgraduates ' innovation capability is a good foundation for the development of various aspects of a country. This paper, taking the competency model as the theoretical basis, analyzes the influencing factors for innovative competency of economics and management postgraduate students. On the basis of analyzing the present situation of innovation capability, taking the economics and management postgraduate students as an example, this paper discusses the influencing factors of innovation capability of postgraduate students from internal and external factors aspects, and can lay the foundation for the further designing of innovation capability index system of graduate students.
\end{abstract}

Keywords — competency model, innovation capability, influencing factors

\section{基于能力素质模型的经济管理类研究生创新能力影响 因素分析}

白冰 郭志琼

江苏师范大学商学院，徐州，江苏，中国

摘 要 随着中国成为创新型经济国家进程的加快, 对于创新人才的培养至关重要。研究生创新能力的培养是一个国家各个方面能 够较好发展的基础。本文以能力素质模型为理论基础, 对研究生创新能力影响因素进行了分析, 以经济管理类研究生为例, 在对其创 新能力的现状分析基础之上, 主要从内部因素和外部因素两个方面对影响经济管理类研究生创新能力的因素进行了分析, 为后续的研 究生创新能力的指标体系的设计奠定基础。

关键词 能力素质模型, 创新能力, 影响因素

\section{1. 引言}

随着中国成为创新型经济国家进程的加快，对于创新 人才的培养至关重要。研究生的创新能力的培养是一个国 家各个方面能够较好发展的基础。而研究生教育处于高等 教育最高层次的, 担负着培养高素质人才和发展科学技术 的双重任务, 是培养高层次创新人才的主渠道。研究生教 育的质量的好坏已经成为衡量一个国家或地区的科学技术
水平高低和专业技术团队整体创新水平高低的重要影响因 素, 这些因素可以直接影响一个国家或地区的政治、经济 以及社会的发展。抓好研究生质量的培养, 是培养高水平 高层次高素质人才的关键, 尤其是研究生创新人才的培养。 因此, 研究生创新能力培养已经逐步成为我国研究生教育 之中的热点问题之一。

本文依据创新人才个人能力素质模型, 以及创新人才

国家社会科学基金教育学项目（No. CIA130172） 
个人能力与科研团队资源能力的匹配关系, 以经管类研究 生为研究对象, 从内部因素和外部因素两个方面对影响经 济管理类研究生创新能力的因素进行了分析, 为后续的研 究生创新能力的指标体系的设计提供支持。

\section{2. 能力素质模型的理论渊源}

“能力素质”也叫“胜任力 (competency) ”, 是 1973 年 哈佛大学的戴维・麦克兰德教授最早提出的, 是从品质和能 力层面论证了个体与岗位工作绩效的关系。他认为个体的 态度、价值观和自我形象, 动机和特质等潜在的深层次特 征, 将某一工作(或组织、文化)中表现优秀者和表现一般者 区分开来, 这些区别特征后来被称作素质。认为素质是决 定工作绩效的持久品质和特征, 也是真正导致员工产生高 绩效的内在动力源泉。它反映的是可以通过不同方式表现 出来的员工的知识、技能、个性与内驱力等。

斯班赛(1993)在 《工作胜任能力》一书中将能力素质定 义为个体具备的某种或某些潜在特质, 这些特质与高绩效 员工的工作表现具有高度的因果关系。

鲍依兹将能力素质定义为一个人所具有的内在的稳定 的特性, 它包括动机、特质、技能、自我印象、社会角色, 以及此人具有的某项知识。

中国学者也对能力素质进行了定义, 认为能力素质是 一个组织中绩效优秀的员工所具备的知识、技能、能力、 人格、动机。结合到具体的岗位或模型, 根据所预测的绩 效标准, 能力素质可以分为门槛类能力素质 (Threshold
Competencies) 和区分类能力素质 (Differentiating Competencies)两类。门槛类能力素质是每个人在工作中必 需的最基本的素质, 是不能区分优秀者与普通者绩效的特 征, 也叫基础能力素质; 区分类能力素质是指能够将优秀 与普通绩效区分的能力素质, 也叫特殊能力素质。

能力素质模型是将能力素质 (职业素养、能力和知识) 按内容、角色或是岗位有机地组合在一起, 职业素养、能 力和知识中的每项内容都会有相关的行为描述, 通过这可 观察、可衡量的行为描述来体现员工对于该项职业素养、 能力和知识的掌握程度。典型的能力素质模型是冰山模型 (Iceberg Competency Model)和洋苾模型。

能力素质模型具有两个核心特点: 一是能力素质是可 以观察和衡量的。二是能力素质的确定及其指标的测量都 必须建立在客观依据之上。本文主要依据能力素质模型, 结合文献分析、研究生拔尖人才的培养目标和培养模式, 初步确定研究生的能力素质构成及其指标体系设计。

\section{3. 基于能力素质模型的研究生创新能力构成}

根据典型的能力素质模型冰山模型 (Iceberg Competency Model), 结合在对研究生能力素质文献分析、 研究生培养目标和培养模式分析的基础上, 经过去除重复 项、合并相似项、去除描述过于泛化的项目等一系列归纳 总结, 结合咨询访谈, 初步提出研究生创新人才需具备的 能力特征。研究生创新人才能力特征至少应具备品质与个 性、知识、技能等个方面:

表 1 基于能力素质模型的研究生创新能力构成

\begin{tabular}{ll}
\hline \multicolumn{1}{c}{ 维度 } & \multicolumn{1}{c}{ 特征项 } \\
\hline 个性品质 & 毅力、责任心、事业心、创新精神、合作精神、发展力 \\
知识 & 理论知识、专业知识、通识知识、环境知识 \\
技能 & 计划、组织、协调、沟通、学习、创新 \\
其他 & 适应能力、综合能力 \\
\hline
\end{tabular}

\section{4. 基于能力素质模型的经管类研究生创新能力现状}

(1) 在个性品质方面, 经管类研究生的创新意识和创 新思维相对较弱。由于经济管理学科自身的特点决定了创 新意识和创新思维在本学科的重要性, 因此, 经管类研究 生的创新意识和创新思维的强弱对经管类研究生的创新能 力提高起着至关重要的作用。经济学中的亚当斯密, 管理 学中的泰罗就是通过创新思维找到了经管类学科的新领 域, 从而推动整个学科的发展。然而, 由于我国在体制与 机制层面的原因, 使得经管类研究生的创新意识和创新思 维较差。
(2) 在知识方面, 经管类研究生缺乏创新所具备的的 综合知识。经济管理学科是一门理论性和实践性结合非常 紧密的学科, 对于研究生来说, 不仅要具体扎实的理论知 识和专业知识, 还需要具体通识知识和环境知识, 才能为 创新奠定基础。理论知识和专业知识是在常规课程学习中 学到的, 而通识知识和环境知识是需要研究生不断的扩展 自身的知识面才能逐步提高和完善的, 因此, 这就需要经 管类研究生在学习自身专业课程的同时, 加强课外其他知 识的学习。

（3）在技能方面, 经管类研究生组织协调的能力相对 
较好。经济管理类学科学习内容大多和实践组织管理有关, 对于如何进行计划、组织、协调、沟通等有着系统全面的 讲解, 因此, 大部分经济管理类研究在此方面受过系统化 的学习和训练, 因此, 经管类研究生在计划、组织、协调、 沟通等方面相比理工类研究生要优秀一些。而计划、组织、 协调、沟通等能力是实现创新的所需要的能力, 尤其是在 科研团队里, 这些技能体现的尤为重要。

（4）其他方面, 经管类研究生创新动机较强, 而创新
成果较少。经管类学科本身是软科学门类, 因此其中枯燥 的理论并不多, 能够激发经管类研究生学习和创新的兴趣。 经管类研究生的自身需求可以通过研究发现经济管理前沿 的理论, 从而进行创新使自身的物质基础需求到自我价值 实现的高级需求都会得到满足, 进而进一步激发研究生创 新动机。由于经管类学科是后兴起的学科, 理论体系形成 滞后于其他理工类学科, 经管类学科研究的方向比较少, 研究的内容比较窄, 导致经管类研究生创新成果较少。

表 2 经管类研究生创新能力现状

\begin{tabular}{ll}
\hline \multicolumn{1}{c}{ 维度 } & \multicolumn{1}{c}{ 现状 } \\
\hline 个性品质 & 创新意识和创新思维相对较弱。 \\
知识 & 缺乏创新所具备的的综合知识 \\
技能 & 组织协调的能力相对较好 \\
其他 & 创新动机较强, 而创新成果较少。 \\
\hline
\end{tabular}

\section{5. 基于能力素质模型的研究生创新能力影响因素分 析}

(1) 内在因素。内在因素是指研究生本身所具有的个 性品质、知识等对于创新能力的影响。我们知道任何事物 的内因是事物发展的根本原因, 决定着事物的性质和发展 方向。因此, 对于研究生的创新能力的影响主要来源于研 究生自身的内部因素。以经济管理类研究生为例, 研究生 需要通过自己的创新能力去研究或开发出具有一定科学价 值的创新成果。经济管理类研究生可以通过内在因素的提 高来提升自身的创新能力, 这是成功进行创新的前提和关 键。从能力素质模型的视角来看, 影响研究生创新能力的 内在因素主要包括个性、品质、知识等方面。

从个性方面来说, 经管类学科需要研究生有发散的思 维和逆向思维, 具备了发散思维和逆向思维的个性, 出现 创新性的成果的概率就会提高。因此, 我们说具有一定的 个性是经济管理类研究生实现创新的重要影响因素。影响 经管类研究生创新能力的个性中不能缺少创新精神。创新 精神是创新能力的灵魂、是创新能力的前提。

从品质方面来说, 研究生自身所具有的品质, 对于成 功实现研究生的创新起到关键性的作用。坚韧的毅力, 持 之以恒的决心是进行科学探索必备的品质。经济管理所要 解决的问题大多是人涉足的领域, 没有现成的样本和经验 可以参考, 经常会面临创新的受挫和失败, 因此, 需要经 济管理类研究生在创新过程中要有不怕失败的精神, 即持 之以恒的毅力。同时, 需要勤奋好学的品质来支撑整个创 新研究的过程, 要不畏研究的辛苦和枯燥创新行动才会更 加及时与准确, 有可能取得最后的成功。

从知识方面来说, 经济管理学科是一门综合性的交叉
学科, 是系统研究经济管理活动的基本规律和一般方法的 科学。除了要求学习者具备扎实的理论知识以外, 更要能 应用相关理论解决实际经济管理的问题, 这就要求经济管 理类的研究生除了具备本学科的专业知识以外, 还要掌握 其他相关学科的知识, 才能综合应用于实践, 实现集成创 新。

(2) 外在因素。外在因素是指影响研究生进行创新活 动的外部环境, 主要包括研究生培养相关的政策、机制及 导师、所处科研团队等。外在因素虽然不是影响研究生实 现创新的关键因素, 但也对研究生创新能力的实现和提升 有着重要的影响。主要表现在以下方面:

在政策机制方面, 国家为了促进研究生创新能力的提 升, 提高研究生的培养水平, 会出台一系列的相关政策措 施来作为保障, 如研究生培养机制改革的各项措施等。这 些政策措施保障了研究生进行创新活动的顺利进行, 有利 于研究生创新能力的培养。但是, 有些滞后的政策措施也 可能对研究生的创新活动产生负面的影响, 如果过多的强 调科研成果的数量, 而忽视科研成果的质量, 导致研究生 创新活动存在急功近利的情况, 这需要政策制定者充分从 鼓励研究生出高水平科研成果的方面去思考如何制定政策 措施, 否则将影响研究生创新活动的进行。

在科研团队方面, 研究生所处的科研团队对研究生的 创新能力有着直接的影响。研究生依托科研团队成长并在 科研团队中发挥作用, 从而提高自身知识水平和创新能力。 如果研究生所在的科研团队是由一些本领域的知名学者和 专家组成, 那么将会对研究生的创新能力提高起到推动作 用, 反之, 如果研究生所在的科研团队总体科研水平不高, 团队合作和创新精神不足, 那么将会削弱研究生本身所具 
有的创新动力, 致使创新能力不高, 即研究生个人能力与 所处科研团队资源能力的匹配关系。因此, 一个好的科研 团队能促使其团队成员的研究生创新能力的提高和创新潜 力的开发。

在导师方面, 研究生导师的指导能力对研究生创新能 力的提高尤为重要。我们知道研究生阶段的学习与本科生 阶段的学习有着本质的区别。导师对于研究生的跟踪指导 是培养研究生的主要方式, 但是, 目前很多高校的研究生 导师平均指导 3-4 名研究生, 而经管类达到 4-5 名之多, 对 于研究生的单独指导严重不足, 而经管类研究生的创新研 究恰恰需要大量时间和精力来完成, 得不到及时有效的指 导, 创新研究受到阻碍。所以, 合理配置导师资源, 优化 导师结构, 将会对研究生创新能力的提高起到促进作用。

\section{6. 结束语}

本文基于能力素质模型, 总结了研究生创新能力构成, 以经济管理类研究生为例, 在对其创新能力的现状分析基 础之上, 阐述了影响研究生创新能力的因素, 主要从内部 因素和外部因素两个方面进行了分析, 为后续的研究生创 新能力的指标体系的设计奠定基础。

\section{参考文献(References)}

[1] McClelland, D. C.. Testing for competence rather than for "intelligence." American psychologist, Vol. 28(1), 1. 1973

[2] Spencer and Spencer, 1993 L.M. Spencer, S.M. Spencer Competence at work models for superior performance John Wiley and Sons Inc, United States of America 1993
[3] William J. Rothwell, John E. Lindholm, Competency identification, modelling and assessment in the US A, International Journal of Training and Development,Vol.3, pp.90-105, 1999.

[4] Boyatzis R. E.. Competencies in the 21st century. Journal of Management Development, Vol 27 (1), 5-12. 2008.

[5] Deng Xiu Quan, Kang Yun Peng, Xi Jun Feng, Bai Bing, Mao Yang $\mathrm{Xu}$. Research on resource competence model of research team in university and its application. Studies in Science of Science. Vol. 30, pp. 102-110, 2012.

[6] Schippmann et al., The practice of competency modeling. Personnel Psychology, Vol 53, 703-740. 2000.

[7] Ma Yibo. Study on innovative ability evaluation system about economics and management graduates. Harbin Engineering University Dissertation.2010.

[8] Bradley, Elizabeth H.; Cherlin, Emily, Adopting a Competency-Based Model; Mapping Curricula and Assessing Student Progress, Journal of Health Administration Education, Vol.25, pp. 37-51, 2008.

[9] Lin Chongde. Ways to cultivate innovation ability from the perspective of the characteristics of creative talent. Scientific Chinese. vol. 9, pp. 18-21, 2011.

[10] Campion, M.A., Fink, A.A., Ruggeberg, B.J., Carr, L., Phillips, G.M., \& Odman, R.B.. Doing competencies well: best practices in competency modeling. Personnel Psychology, Vol.64, 225-262.2011. 